\title{
Efficient Data Collection for Wireless Sensor Network using Virtual Grid based Clustering
}

\author{
Pradeep Rao K. B. \\ PG Scholar, Dept. of CSE \\ NMAMIT, Nitte, India
}

\author{
Ravi B. \\ Research Scholar, \\ Dept. of CSE \\ NMAMIT, Nitte, India.
}

\author{
Kiran Kumari Patil \\ Professor, School of \\ Computing and IT, \\ Reva University, India.
}

\begin{abstract}
Mobile sink based data gathering for improving network lifetime in Wireless Sensor Networks (WSNs) has been extensively studied in literature. Mobile sinks are useful in mitigating energy-hole problem that can occur due uneven energy consumption among sensor nodes. However, the approach introduces new challenges during data delivery due to continuous change in the sink location. For efficient data delivery, sensor nodes need to readjust their routes toward the latest sink location with minimal overhead in large networks. In this paper we propose Virtual Grid based Data Collection (VGDC) scheme which allows sensor nodes to deliver data with minimal route reconstruction overhead toward the latest location of mobile sink. We evaluate and analyze the performance of the approach using simulations. Simulation results demonstrate that VGDC scheme reduces overall energy consumption of the network and enhances network lifetime.
\end{abstract}

\section{Keywords}

Wireless Sensor Network, Mobile sink, Virtual Grid, Data Gathering, Route Adjustment

\section{INTRODUCTION}

Wireless sensor network (WSN) consists of large number of sensor nodes widely dispersed over large area for sensing various physical and environmental conditions such as temperature, sound, pollution levels, humidity, wind speed and pressure [2]. Each sensor node is battery operated, having limited memory and processing capability. For many applications, sensor nodes in a WSN are deployed in a remote hostile environment, which makes them unattended devices. Replacing their battery in such applications is difficult or not feasible. Therefore, developing techniques for efficiently utilizing battery power of sensor nodes has been a critical area of research. All sensor nodes in a WSN eventually deliver their measurements to a central base station or sink. In the recent past, mobile sink based data gathering has been extensively investigated by researchers for enhancing the network lifetime.

In static sink WSN model, readings of sensor nodes are routed to the sink via multi-hop forwarding. Since the sink is stationary, this method of data gathering creates bottlenecks in the WSN, particularly in the area around the sink. That is, sensor nodes near the sink deplete their battery power early compared to the sensor nodes that are farther away from sink. This causes uneven distribution of sensor node's energy consumption in the WSN. This finally results in network partition making the sink impossible to receive from its nearby nodes. In other words "energy-hole" or "hot spot" is created around the sink. These energy-holes are one of major causes for decreased network lifetime in WSN with stationary sink.
To mitigate energy-hole problem and thus improve network lifetime, data gathering approaches using mobile sink have been proposed in literature. These approaches allow a mobile sink to travel along the sensor field and gather data from the nearby sensors nodes at regular intervals. Sink mobility in WSN helps in uniformly distributing the energy expenditure of sensor nodes, and hence improves overall network lifetime of WSN. In addition, it helps address the network connectivity issues in sparse networks. However, sink mobility brings new challenges for sensor nodes in delivering sensor data to the mobile sink. Since network topology changes continuously as sink keeps changing its locations. A major challenge here is identifying the current location of the sink so that sensor nodes can route their data to the correct location. An efficient mechanism is therefore required in order to update sensor nodes with the current sink location so that sensor nodes can correctly construct data delivery route toward the sink. Since energy is a scarce resource in WSN, regular transmission of the location update will affect the overall lifetime of the network. Furthermore, sensor nodes require a mechanism that allows them to cooperatively route their data without consuming much energy.

In this paper, we propose Virtual Grid based Data Collection (VGDC) scheme for regular data collection from a WSN. VGDC scheme uses the method similar to the one used in VGDRA [1]. Initially, the entire sensor filed is partitioned into a virtual grid made up of $\mathrm{K}$ equal sized cells. Each cell is assigned a cell-header to which all other sensor nodes within the cell send their data. These cell-headers are responsible for cooperatively forwarding the data to the current location of the mobile sink. As in VGDRA scheme, sensor nodes dynamically readjust their route toward the latest sink location with minimal overhead. In VGDRA, the mobile sink travels along the sensor field periphery and gathers data from border line cell-headers and all other cell-headers forward to this cellheader. In large networks with hundreds of nodes, this puts a heavy data load on the border-line cell-header. To reduce this data load, the proposed scheme routes data from sensor nodes to more than one cluster-head instead of a single cluster-head. To accomplish this, we move the sink in a controlled manner along a fixed path inside the sensor field. In addition to minimizing the load, the approach improves the data delivery performance and reduces the overall energy consumption in WSN.

We organize the paper as follows: Section 2 briefly reviews related work. Section 3 provides detailed description of VGDC scheme. Simulation results and performance analysis are highlighted in section 4 . Finally, section 5 concludes the paper.

\section{RELATED WORK}

Utilizing mobile sink in a WSN for data gathering is a critical area of research and has been extensively studied by many 
researchers with the goal of improving the network lifetime. Sink based data collection schemes are based on either controlled sink mobility or uncontrolled sink mobility [4]. In the controlled sink mobility schemes, sink movement is governed by several parameters such as speed, direction, data collection latency, sensor node's residual energy etc. In the later approach, the sink moves randomly without any restrictions on speed and directions. In such a scheme, the mobile sink communicates with its nearest node for direct data transfer. As the sink is uncontrollable, predicting the next position of the sink is difficult. In the controlled sink mobility schemes, network or observer of the network controls the movement of the mobile sink. Below we briefly describe some of the approaches.

R. C. Shah et al. [5] proposed an architecture made up of three tiers for collection of data from sparse sensor networks. The approach used mobile entities, called MULEs, that are responsible for data collection from sensor nodes. When MULEs appear within the range of sensor nodes, data is capture and buffered, and then forwarded to wired access points. MULE architecture consists of three tiers, namely top tier, middle tier and bottom tier. Top tier is consists of access points which collect data from MULEs and sends it to WAN. Middle tier consists of mobile transport agents, which are used to provide connectivity to the network. Bottom tier contains randomly scattered sensor nodes. Heinzelman et al. [6] proposed Low-Energy Adaptive Clustering Hierarchy (LEACH) protocol to distribute energy uniformly across the network. In LEACH, nodes are grouped to form clusters, where each cluster contains cluster head and member nodes. Member nodes deliver data to their cluster head. Cluster heads combine the data and finally deliver it to the base station. Cluster head is chosen at random and changed in each round. Each round begins with a set up phase during which nodes are grouped to form cluster. Set up phase is followed by steady state phase during which cluster head send data to the base station.

Erman et al. [7] proposed a hexagonal based grid structure for timely delivery of real-time data to the mobile sink. Query and data rendezvous lines are defined by hexagonal cell-based data dissemination (HexDD) protocol to prevent unnecessary transmission of sink's queries. Border line nodes receive data from sensor nodes and propagate the data to the center cell. Border line nodes store and replicate the received data. Queries of the sink are relayed to centre cell and border line cell-headers deliver data to the sink. However, this scheme causes sensor nodes to consume more energy when sink moves at higher speed. Oh et al. [8] proposed Backbone-based Virtual Infrastructure (BVI) scheme for distribution of data to the mobile sink. BVI scheme employs single level multi-hop clustering for minimizing the network overhead. Cluster heads are chosen based on the residual energy of the sensor nodes. A node with higher residual energy has more chance to become a cluster head. In order to find the sink node position, a cluster head is chosen as the root of the data forwarding tree. Route readjustment heavily depends upon the root node and early energy depletion of the root node causes decrease in the network lifetime.

Chen et al. [9] presented a novel Virtual Circle Combined Straight Routing (VCCSR) algorithm, which is a structure based routing with a virtual backbone. Virtual backbone network contains a group of cluster heads. As mobile sink travels across the sensor field for data collection, VCCSR constructs dynamic routing tree on the virtual backbone. VCCSR follows certain updating rules for the virtual structure to adjust the routes effectively. Kinalis et al. [10] introduced biased sink mobility scheme in which stopping time of the sink depends upon network traffic. The sink stopping time is relatively less at sensor regions where traffic is low and more at regions where traffic is high.

Khan et al. proposed Virtual Grid based Dynamic Routes Adjustment (VGDRA) scheme for maintaining routes toward the latest location of the sink. VGDRA divides sensing area into equally sized cells based on the number of sensor nodes in the network. From each cell, a sensor node is selected as the cell-header and member nodes relay data to the respective cell-header. Mobile sink travels around the periphery of the sensor field and collects data from border line cell-headers. The cell-header nearest to the mobile sink, called Originating Cell Header $(\mathrm{OCH})$, is responsible forwarding the data from other cluster-heads to the mobile sink. VGDRA scheme follows set of routes readjustment rules which allows the cellheaders to adjust their data delivery routes toward the current sink location. In the proposed scheme, the mobile sink moves inside the sensing area along a fixed path so as to minimize the route readjustment overhead and energy consumption rate for sensor nodes. In contrast to VGDRA, VGDC scheme communicates with more than one cell-header instead of one cell-header. Data from other cell-headers is routed toward these cell-headers, and thus no individual cell-header has the burden of receiving traffic load from several cluster headers. Simulation results demonstrate that this approach results in better distribution of energy consumption among sensor nodes.

\section{VGDC SCHEME}

In this section we describe the proposed scheme in detail. Initially, a virtual grid is constructed by partitioning the sensor field into an appropriate number of cells. We then use a method that enables sensor nodes to deliver data to the latest mobile sink location. The scheme uses controlled sink mobility to make the sink travel inside the sensor field for gathering sensor data.

\subsection{Assumptions}

The assumptions made in this paper are:

- Sensor nodes are randomly deployed in the sensor field.

- Mobile sink has unlimited energy reserve.

- Every sensor node has sufficient buffer space.

- Sink node travels with a constant speed.

- WSN consists of homogeneous sensor nodes and all nodes are aware of their locations.

\subsection{Virtual Grid Construction}

The entire sensor field is partitioned into equal sized cells as shown in Fig. 1 The optimal number of cells according to the heuristics used in LEACH [6], TEEN [11], and APTEEN [12] is $5 \%$ of the total number of sensor nodes deployed in the network. If $\mathrm{N}$ number of nodes are randomly scattered in the sensor field, Equation 1 gives the number of equally sized cells for a maximum of 6,12 , and 20 nodes. Each cell contains randomly distributed sensor nodes.

$$
K=\left\{\begin{array}{lr}
4 & N * 0.05 \leq 6 \\
9 & 6<N * 0.05 \leq 12 \\
16 & 12<N * 0.05 \leq 20
\end{array}\right.
$$




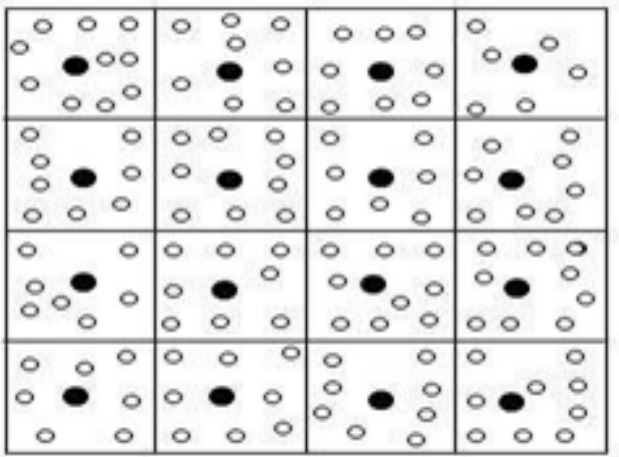

Fig. 1: Partitioning of sensor field into cells

After partitioning the network area into an appropriate number of cells, a cell-header is chosen from each cell. For each cell, the sensor node nearest to the mid-point of the cell is chosen as the cell-header. Threshold based cell-header selection is used to minimize the communication cost while choosing a cell-header. A threshold distance is chosen from the mid-point of the cell, and nodes under this threshold distance are candidate nodes for the cell-header. The threshold distance is gradually increased if no node falls under the threshold distance. Any border cell-header can have maximum of 3 adjacent cell-headers whereas any non-border cell-header can have maximum of 4 adjacent cell-headers. A cell-header communicates with its adjacent cell-header via gateway nodes. The gateway nodes and the cell-headers constitute the virtual backbone structure.

\subsection{Data Delivery Mechanism}

Before data gathering begins, a fixed rectangular sink path is computed such that the path covers almost half the area of the network area. Fig. 2 shows the initial sink position and travelling path for a network with 16 cells. The figure also shows the direction of sink movement. As the sink travels along the fixed path within the sensor field, it maintains communication with the nearest cell headers. We call these cell-headers as Originating Cell Headers (OCHs). These OCHs collect the current sink location information from the sink and propagate it to the rest of the cell-headers. In this way, all the cell-headers update the latest sink location entry. Cell-headers upon receiving the location information adjust their data delivery route toward the current sink location. A cell-header always identifies the nearest $\mathrm{OCH}$ and readjusts its route to that $\mathrm{OCH}$. A cell-header maintains this route information in its routing table until it receives new sink location information. After data is gathered from OCHs, the mobile sink locates new OCHs and updates them and sensor nodes with the new location information. To reduce route construction overhead, existing route is re-adjusted by the cluster-heads using readjustment procedure which we describe below.

Upon detecting the mobile sink, an OCH checks to see if the sink is already its next-hop in its routing entry. If the next-hop is already the sink, the location information of the sink is not propagated by the $\mathrm{OCH}$. Otherwise, the $\mathrm{OCH}$ sets the mobile sink as its next hop and shares the location update with the previous $\mathrm{OCH}$ and its downstream adjacent cluster-head. The previous $\mathrm{OCH}$ upon receiving the location information updates its routing entry by setting the previous $\mathrm{OCH}$ as the next-hop toward the mobile sink. The downstream clusterhead upon receiving the location update checks to see if sender of the location update is the previous next-hop. If it is not the previous next-hop, then the new sender is set as its next-hop and the location information propagated to the next downstream cluster-head. Otherwise, the location information is simply dropped. This procedure is repeated till all the downstream cluster-heads update their next-hop information toward the latest location of the mobile sink.
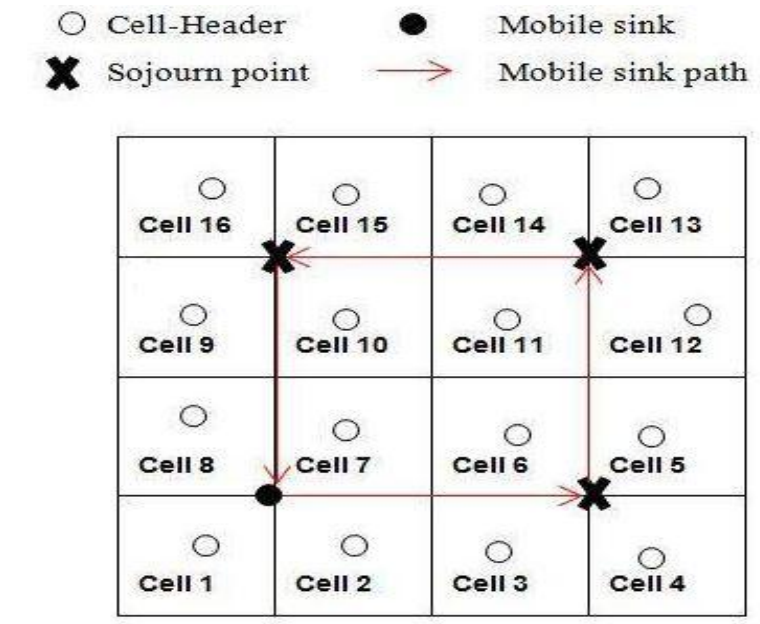

Fig. 2: Path construction for the mobile sink

Fig. 3 shows the route readjustment process in VGDC scheme, where the mobile sink sends its current location information to the cell-headers 2, 3, 4 and 5. The location information is then propagated to the rest of the cell-headers. With the received location update, cluster-heads readjust their data delivery path toward the latest sink location.
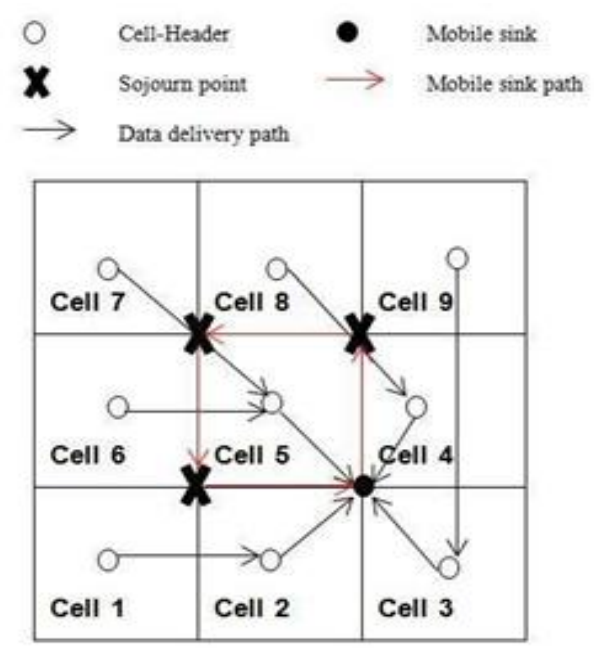

Fig. 3: Delivery of data in VGDC scheme

Algorithm: VGDC

1. Construct the virtual grid by partitioning the sensor field into equally sized cells according to Equation 1.

2. Choose a cell-header from each cell.

3. Compute a fixed travelling path for the sink such that the area covered by the path is almost half the application area.

4. Start moving the sink

5. Locate the nearest $\mathrm{OCHs}$ and pass them the current sink location information.

6. Apply the route readjustment procedure to propagate the location information to all cell- 
headers and readjust the existing route to reflect the fresh route toward the sink.

7. Collect data and go to step 4 .

\section{RESULTS AND DISCUSSIONS}

Using simulations we evaluate the performance of the proposed scheme for different network topologies. We used ns2 simulator to set up the network and $\mathrm{C}++$ to implement route readjustment mechanism. Network topologies, with varying number of nodes $(100,150,200,250,300)$ that are randomly deployed within a sensor field of $500 \times 500$, are considered. All sensor nodes have an initial energy of 100J. The mobile sink travels with a constant speed of $5 \mathrm{~m} / \mathrm{s}$. Each sensor node produces a data of size 500 bytes at regular intervals. Table 1 slows the lists of various parameters used in the experiments. Network lifetime is defined as the time until the first node depletes $80 \%$ of the initial energy. We measure the average energy consumed by the sensor nodes and consider this for comparing with VGDRA.

Table 1. Simulation Parameters

\begin{tabular}{|l|l|}
\hline \multicolumn{1}{|c|}{ Parameter } & \multicolumn{1}{c|}{ Value } \\
\hline Simulation area & $500 \mathrm{~m} \times 500 \mathrm{~m}$ \\
\hline Number of sensor nodes & $150,200,250,300$ \\
\hline Mobile sink speed & $5 \mathrm{~m} / \mathrm{s}$ \\
\hline Communication range & $50 \mathrm{~m}$ \\
\hline Packet length & 512 bytes \\
\hline Sensor node's energy & $100 \mathrm{~J}$ \\
\hline
\end{tabular}

Fig. 4 shows the network lifetime for networks with different number of sensor nodes. As the figure shows, VGDC improves the network lifetime for large networks. This is because of the reduced distance that data packets have to traverse from cluster-heads to the sink. In VGDRA the sink moves at the periphery of the application area due to which data packet takes more hops through the routing path to reach the mobile sink. In VGDC the sink is made to travel along the computed path inside the sensor field.

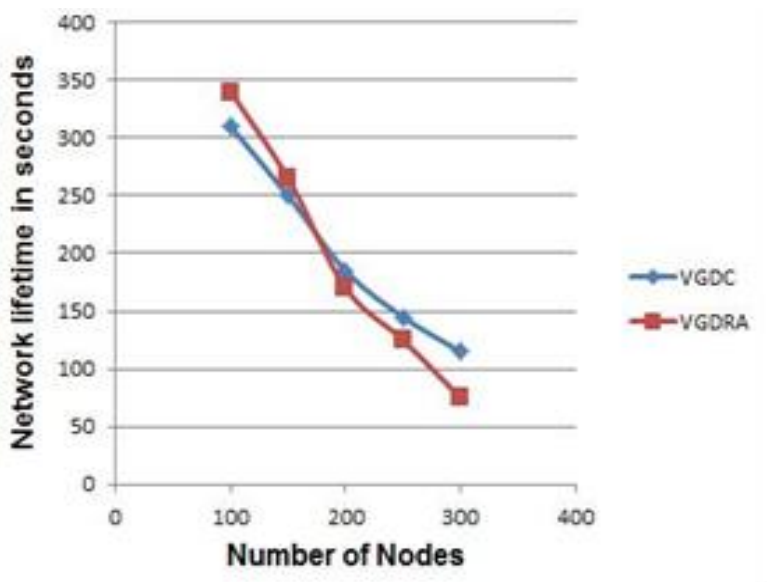

Fig. 4. Network lifetime vs. number of nodes

Fig. 5, 6, 7 and 8 depict the average energy consumed by sensor nodes for different topologies. We measured per round energy consumption for each network topology. We allow the mobile sink to complete 6 rounds of data collection and measure energy consumption for each round. Both the approaches consume the same amount of energy in the initial
3 rounds for 150 nodes. As the sink makes more rounds, there is a considerable reduction in the energy consumption in VGDC as compared with VGDRA. For higher network sizes, VGDC shows a small increase in the performance for initial rounds which indicates that the approach scales well in terms of energy consumption. The major reason for this is the ability of the scheme to balance the data load among cluster-heads, which results in reduced energy consumption among sensor nodes. With the proposed method, the reduced route readjustment overhead also contributes to the goal of minimizing the overall energy expenditure of the network. Furthermore, the method balances multi-hop routes between the cluster heads and mobile sink.

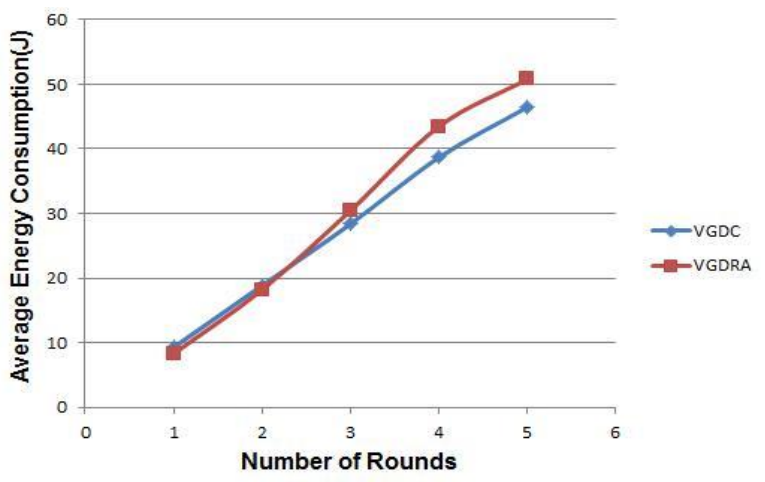

Fig. 5: Per round average energy consumption for 150 nodes.

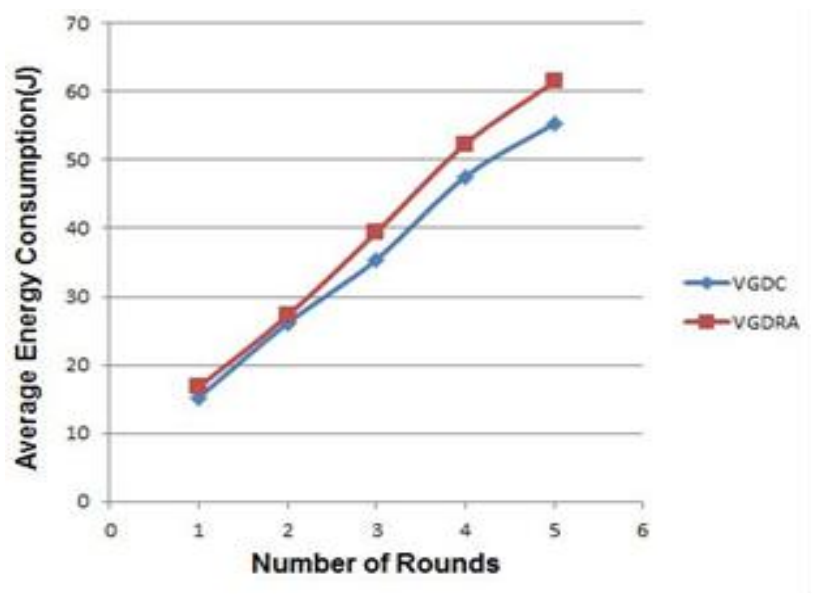

Fig. 6. Per round average energy consumption for 200 nodes

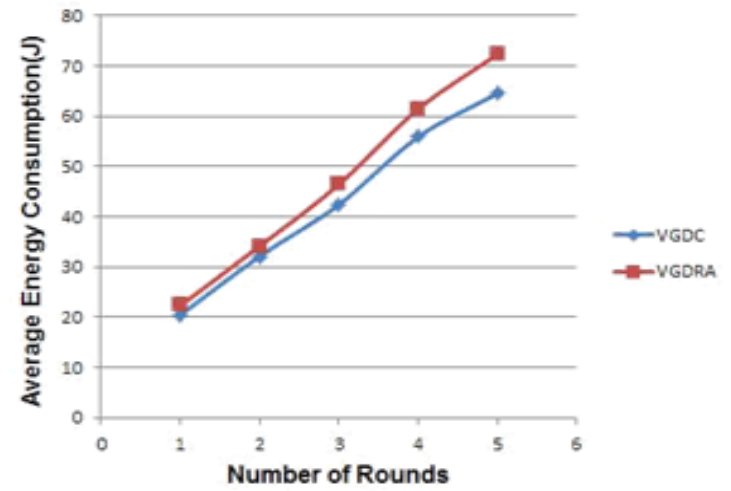

Fig. 7. Per round average energy consumption for 250 nodes 


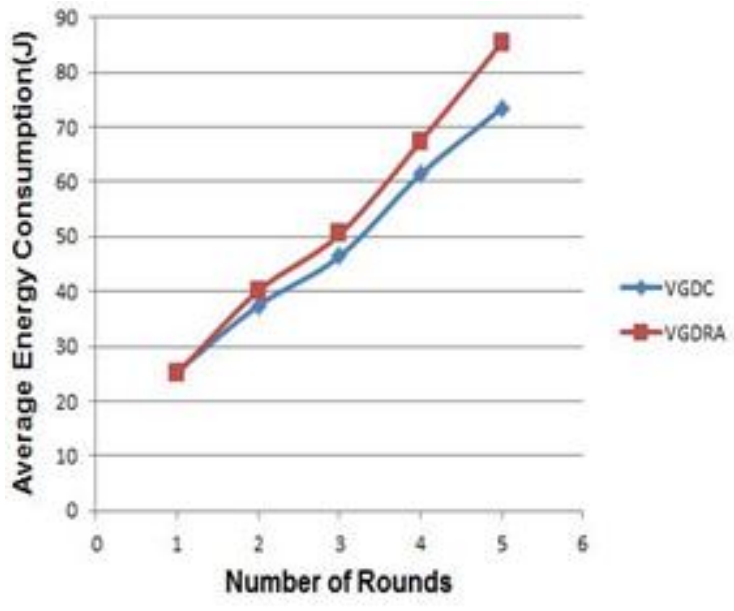

Fig. 8. Per round average energy consumption for 300 nodes

\section{CONCLUSIONS}

In this paper, we proposed VGDC scheme that utilizes a mobile sink for data gathering in a WSN with the aim to minimize route construction overhead and improve the network life. The aspect of mobile sink based data gathering in a WSN is considered a very important and challenging one. The proposed method attempts to address the problems that can occur during data gathering from a WSN using mobile sink. The method is based on constructing a virtual backbone by partitioning the entire sensor field into equal sized cells, where each cell has randomly scattered sensor nodes. Each cell is assigned a cell-header which is responsible for gathering the data from its member nodes and forwarding it to the latest sink location. A fixed path for mobile sink is computed with the aim to minimize the route readjustment overhead and improve the network lifetime. During data collection, cluster-heads are updated with the latest location information of the sink so as to enable them to readjust delivery paths toward the current sink location. The sink passes its location information to the nearest cluster-heads which propagates the information to rest of the cluster-heads. We compared the performance of VGDC with VGDRA in terms of network lifetime and average energy consumption using simulations .The results demonstrate VGDC scheme shows better performance in large networks. In future work, we plan to evaluate the results of using different sink travelling paths, speed and data rates for heterogeneous WSNs.

\section{REFERENCES}

[1] A. W. Khan, A. H. Abdullah, M. A. Razzaque and J. I. Bangash, "VGDRA : A Virtual Grid based Dynamic Routes adjustment Scheme for Mobile Sink based Wireless Sensor Networks," Sensors, vol. 15, no. 1, pp. 1-9, Jan. 2014.
[2] I. F. Akyildiz et al., "A survey on sensor networks", IEEE Communications, vol. 40, pp. 102-114, Aug. 2002.

[3] A. Somasundara, A. Ramamoorthy and M. Srivatsava, "Mobile Element Scheduling for Efficient Data Collection in Wireless Sensor Networks with Dynamic Deadlines," Proc. 25 $5^{\text {th }}$ IEEE Int'l Real-Time Systems Symp. (RTSS), pp. 296-305, 2004.

[4] A. W. Khan, A. H. Abdullah, M. H. Anisi, and J. I. Bangash, "A comprehensive study of data collection schemes using mobile sinks in wireless sensor networks," Sensors, vol. 14, no. 2, pp. 2510-2548, 2014.

[5] R. C. Shah, S. Roy, S. Jain, and W. Brunette, "Data MULEs: Modeling and analysis of a three-tier architecture for sparse sensor networks," in Ad Hoc Netw., vol. 1, pp. 215-233, 2013.

[6] W. B. Heinzelman, A. P. Chandrakasan, S. Member, and H. Balakrishnan, "An application-specific protocol architecture for wireless microsensor networks," IEEE Trans. Wireless Commun., vol. 1, no. 4, pp. 660-670, Oct. 2002

[7] A. Erman, A. Dilo, and P. Havinga, "A virtual infrastructure based on honeycomb tessellation for data dissemination in multi-sink mobile wireless sensor networks," EURASIP J. Wireless Commun. Netw., vol. 2012, no. 17, pp. 1-54, 2012.

[8] S. Oh, E. Lee, S. Park, J. Jung, and S. H. Kim, "Communication scheme to support sink mobility in multi-hop clustered wireless sensor networks," in Proc. 24th IEEE Int. Conf. Adv. Inf. Netw. Appl. pp. 866872, Apr. 2010

[9] T. S. Chen, H. W. Tsai, Y. H. Chang, and T. C. Chen, "Geographic convergecast using mobile sink in wireless sensor networks," Comput. Commun., vol. 36, no. 4, pp. 445-458, Feb. 2013.

[10] A. Kinalis, S. Nikoletseas, D. Patroumpa, and J. Rolim, "Biased sink mobility with adaptive stop times for low latency data collection in sensor networks," Inf. Fusion, vol.15,pp.56-63,Jan.201

[11] A. Manjeshwar and D. P. Agrawal, "TEEN: A routing protocol for enhanced efficiency in wireless sensor networks," in Proc. $15^{\text {th }}$ Int. Parallel Distrib. Process. Symp. (IPDPS), vol. 1. Apr. 2000, pp. 2009-2015.

[12] A. Manjeshwar and D. P. Agrawal, "APTEEN: A hybrid protocol for efficient routing and comprehensive information retrieval in wireless," in Proc. 16th Int. Parallel Distrib. Process. Symp., 2002, p. 48. 